DANNY MILLER

HEC Montréal, Canada (Qc)

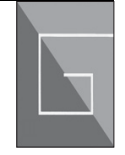

\title{
La dernière décennie (ou presque) de mon travail $^{1}$
}

$\mathbf{J}$

ean-Philippe Denis m'a demandé de décrire mes récentes recherches pour mettre à jour le chapitre qu'il a généreusement écrit à propos de mon travail. Je le fais en sachant qu'un auteur est son représentant le plus partial.

$\mathrm{Au}$ cours des douze dernières années, mon «agitation académique » a pris de nombreuses formes. Une grande partie de la recherche qui en résulte fait ressortir ce que je considère comme un problème social ou une opportunité d'amélioration. Bien qu'un grand nombre de contributions commencent par une position critique, elles proposent tout de même des suggestions positives. On recense différentes catégories caractérisant la recherche :

1. Note rédigée par Danny Miller en février 2018 et extraite de Denis J.-P. (2020), chapitre XII. « Danny Miller. L'approche configurationnelle : une réponse aux paradoxes de la stratégie ? », Les grands auteurs en stratégie, T. Loilier et A. Tellier (dir.), 2 édition, Caen, Editions EMS, p. 219-243 (traduction Alexandra Burlaud). La version publiée ici a été revue par l'auteur et la bibliographie actualisée. 
- Les critiques à l'égard du court-termisme des entreprises cotées en Bourse qui nous ont amenés à étudier une forme négligée d'organisation : les entreprises familiales. - Les critiques du court-termisme managérial qui identifient l'impact de la formation en management sur l'opportunisme des cadres.

- La recherche de modes relationnels à long terme de la gestion des parties prenantes de l'entreprise et d'un management « soutenable».

- La faiblesse engendrant la force des individus et des entreprises désavantagées/ défavorisées.

- Le réexamen de l'approche de la configuration (Configuration approach) (incluant une critique de l'orientation entrepreneuriale).

J'aborderai brièvement chacun de ces thèmes tout en ne mettant l'accent que sur certaines publications.

\section{I - L'ENTREPRISE FAMILIALE : CONTRE LE COURT-TERMINISME ORGANISATIONNEL}

Les histoires abondent dans la presse au sujet de cadres supérieurs qui deviennent riches alors que leur entreprise, leurs employés, leurs parties prenantes, leur environnement naturel s'effondrent. Il devait surement y avoir une alternative. Nous nous sommes intéressés à l'entreprise familiale non pas parce qu'il s'agissait de la forme d'organisation la plus courante dans le monde ou parce qu'elle avait été ignorée par les académiques et les business schools mais parce que nous espérions qu'elle pourrait éviter le courttermisme destructeur de nombreuses sociétés cotées en Bourse ${ }^{2}$.

Nous avons découvert que les entreprises familiales se portaient aussi bien que les autres lorsqu'elles étaient cotées en Bourse (Miller et al., 2007) et qu'elles affichaient souvent un résultat supérieur lorsqu'elles n'ouvraient pas leur capital (Miller et al., 2008). Cependant, celles qui ont réussies ont investi à long terme, en gérant non pas en fonction des bénéfices trimestriels, mais de façon durable, et elles ont surpassé leurs homologues non familiaux (Cho et al., 2018). Les meilleures d'entre elles étaient des intendantes (stewards) : elles ont beaucoup investi dans la formation et la sélection des employés, dans les avantages sociaux du personnel et dans l'enrichissement des emplois. Elles ont également régulièrement renouvelé leur marché des produits, privilégié les relations à long terme avec les clients et ont fait preuve d'une charité inhabituelle à l'égard de leurs communautés (Le BretonMiller et Miller, 2009). Ceci était le cas pour des entreprises familiales anciennes (longlived), publiques et privées, américaines, européennes et asiatiques, dans une grande variété d'industries (Miller et al., 2007, 2008, 2009 ; Miller et Le Breton-Miller, 2005) notamment dans les entreprises dirigées par des couples mariés et également là où il $\mathrm{y}$ avait des vides institutionnels nationaux (Amore et al., 2017 ; Miller et al., 2009).

\footnotetext{
2. Notre douzaine d'études a porté sur de grandes entreprises et de PE familiales quelles soient privées ou cotées en Bourse en Amérique du Nord, en Europe et en Asie. Nous avons étudié la performance en termes de durabilité, de rentabilité, d'évaluation du marché et de survie. Nous avons examiné une myriade de formes de gouvernance au sein de la structure de propriété, la composition du conseil d'administration, la gestion familiale de toutes sortes (de couples mariés aux co-exécutifs, aux cadres héritiers et aux managers externes), nous avons exploré l'innovation, l'internationalisation, les pratiques en matière de ressources humaines et autres.
} 
Toutefois, cette orientation à long terme était moins courante dans les entreprises dirigées par des héritiers, celles qui adoptent des modèles fondés sur de faibles coûts et l'efficacité, et celles qui opèrent dans un environnement institutionnel dominé par des logiques de marché prédatrices ou des logiques familiales autocentrées (Miller et al., 2017).

\section{II - VALEURS MANAGÉRIALES ET MORALITÉ : ÉVALUER LE COURT-TERMISME MANAGÉRIAL}

Les business schools se concentrent sans relâche sur une représentation économique de l'homme : opportuniste, égoïste, avide et indigne de confiance. Le point de vue adopté est celui d'un P-DG ou d'un propriétaire souhaitant tirerun profit personnel. Les parties prenantes telles que les employés, les fournisseurs et les clients sont considérées comme des instruments d'aide dans ce processus. La théorie de l'agence et l'économie des coûts de transaction admettent ces incitations pécuniaires et proposent la surveillance (monitoring) comme moyen d'alignement des intérêts égoïstes dans un climat de méfiance et d'opportunisme (Miller et $\mathrm{Xu}, 2019$ ).

Plusieurs thèmes de notre recherche explorent les conséquences de cette vision prédatrice du monde. Premièrement, nos études sur les P-DG détenant un MBA (Miller et Xu, 2016, 2019). Dans deux études avec de larges échantillons d'entreprises américaines, nous avons constaté que les P-DG titulaires d'un MBA étaient plus susceptibles que les autres P-DG à poursuivre des stratégies opportunistes à court terme comme une gestion fallacieuse des bénéfices, la réduction de la $R \& D$ et des acquisitions dangereuses, afin de gonfler le résultat net et croître rapidement.
Bien que les bénéfices aient d'abord augmenté, les valorisations boursières des entreprises ont par la suite chuté précipitamment à mesure que les entreprises s'affaiblissaient. Ironiquement, au cours de ces intervalles, les P-DG détenant un MBA ont réussi à obtenir des augmentations de rémunération beaucoup plus importantes que leurs homologues.

Par ailleurs, en abordant notre propre métier de chercheur avec un regard critique, nous avons commencé à étudier l'aliénation du travail chez les chercheurs en gestion, constatant que l'esprit de compétition, l'opportunisme et les pratiques égoïstes nuisent à la communauté des chercheurs en gestion, surtout en Amérique (Le BretonMiller et Miller, 2018).

\section{III - VALEURS MANAGÉRIALES ET MORALITÉ : COMBATTRE LE COURT-TERMISME MANAGÉRIAL}

Nous explorons, à travers cette thématique, comment certaines entreprises ont évité le court-termisme et ont adopté des stratégies plus durables au service de toutes les parties prenantes (Le Breton-Miller et Miller, 2020). Réagissant à la critique de Max Weber à l'égard des chefs d'entreprise égoïstes et recherchant l'intérêt pécuniaire, nous avons recherché des entreprises qui atteignent des objectifs sociaux à long terme tels que le progrès scientifique et le bien-être social, souvent fondés sur des valeurs éthiques profondes. Ces entreprises adoptent une « responsabilité fondée sur des valeurs » et représentent une source d'espoir pour l'avenir (Le Breton-Miller et Miller, 2020).

Nous avons également examiné des solutions alternatives à la gestion à court terme dans des entreprises asiatiques qui ont 
excellé en combinant les valeurs de l'Est et de l'Ouest (Chen et Miller, 2010, 2011). De nombreuses entreprises en Corée et à Taïwan ont adopté une forme de gestion « relationnelle» dans laquelle elles établissent des relations à long terme avec leurs employés, leurs fournisseurs et leurs clients, fondées sur la loyauté et la confiance réciproque (Miller et al., 2009 ; Chen et Miller, 2010, 2011). Ces entreprises ont réussi à combiner les pratiques méritocratiques de l'Occident avec les conventions plus relationnelles de l'Orient, ce qui leur a permis de mieux réussir dans leurs efforts de renouvellement commercial, d'expansion internationale et de croissance durable.

Poursuivant un thème similaire, Miller et Sardais (2011), dans une critique de la littérature de l'agence, discutaient de la façon dont certains gestionnaires ont agi en tant «qu'agents providentiels » (angel agents), en mettant en place des pratiques durables au sein de leur entreprise, dans le traitement de leurs employés et de l'environnement, tout en luttant avec diligence contre les actionnaires impatients.

\section{IV - LA FAIBLESSE DANS}

\section{LA FORCE - ET VICE VERSA : QUELQUES LIENS PARADOXAUX}

Certains de nos articles récents ont adopté, sinon un point de vue à contre-courant, du moins un point de vue paradoxal. L'un des thèmes est que la faiblesse mène souvent à la force et vice versa. Un premier indice sur cette idée est apparu dans The Icarus Paradox (Miller, 1990), où des entreprises prospères sont devenues des caricatures extrêmes de leur propre personne et sont tombées dans l'oubli. Nous avons confirmé cette théorie en démontrant les vulnérabilités associées aux ressources de l'entreprise (Le Breton-Miller et Miller, 2015).

Nous avons commencé à nous demander si l'inverse pouvait également être vrai, c'està-dire si le désavantage initial était associé à un avantage subséquent. Par exemple, la littérature neurologique a suggéré d'importants avantages sur le plan de la perception et de la reconnaissance des formes (pattern recognition), ainsi qu'une orientation vers l'action, chez les dyslexiques, ce qui, selon moi, explique en partie leur nombre disproportionné chez les entrepreneurs performants (Miller, 2011a).

En élargissant cette vision «positive » $\mathrm{du}$ handicap, nous avons constaté qu'un pourcentage élevé d'entrepreneurs dans de nombreuses régions du monde présentaient d'autres types importants de désavantages : difficultés d'apprentissage, handicaps physiques, pauvreté, manque de compétences professionnelles, traumatisme causés par la guerre et aliénation culturelle. Nous avons soutenu que les défis et les expériences de ces individus ont inculqué en eux une force de caractère, des aptitudes sociales, des orientations motivationnelles et des points de vue originaux et créatifs qui ont fait d'eux de meilleurs entrepreneurs (Miller et Le Breton-Miller, 2017).

\section{V - EXAMEN DE LA NOUVELLE APPROCHE DE LA CONFIGURATION : OÙ SONT LES CONFIGURATIONS ?}

Ayant plaidé en faveur de l'importance des configurations et de la taxonomie comme fondements de la Mid-range Theory en gestion au début de ma carrière, j'ai réexaminé la question dans nos études sur les entreprises familiales et leurs stratégies. 
Nous avons utilisé la configuration pour mieux comprendre comment différents types d'entreprises familiales ont orchestré leurs éléments de stratégie et d'organisation pour atteindre une performance durable (Miller et Le Breton-Miller, 2005, 2006). Plus récemment, il est également apparu clairement qu'il y avait un renouveau continu de la notion de configuration comme moyen de mieux différencier les organisations et leur fonctionnement (e.g. Misangyi et Acharya, 2014). De nombreux chercheurs utilisaient l'analyse de cas qualitative ou AQC - une technique fondée sur la logique booléenne - pour déterminer si certains éléments de la stratégie ou de l'organisation étaient complémentaires ou substituables en ce qui concerne certains résultats. Malheureusement, il n'y a pas eu de riches caractérisations des configurations ou de leurs processus et une trop grande dépendance à l'égard de simples tests de théories. Dans Miller et al. (2018), j'ai proposé un moyen de mélanger le meilleur de l'ancienne et de la nouvelle approche de la configuration et suggéré dans quel cas l'une serait préférable à l'autre.

J'ai également revu mon article de 1983 sur l'orientation entrepreneuriale, suggérant que malgré sa popularité, son message central s'était perdu : trop peu d'études subséquentes ont établi les distinctions de configurations nécessaires pour faire des prédictions contextualisées (Miller, 2011b). En jetant un regard rétrospectif sur cette liste de projets quelque peu épuisante, je dois dire que j'ai énormément apprécié mes partenariats avec tant de collègues impliqués dans ce travail et j'espère poursuivre sur cette voie dans les années à venir.

\section{BIBLIOGRAPHIE}

Amore M., Miller D., Le Breton-Miller I. et Corbetta G. (2017). "For love and money: Marital leadership in family firms", Journal of Corporate Finance, vol. 46, p. 461-476.

Chen M.-J. et Miller D. (2011). "The relational perspective as a business mindset: Lessons from East and West”, Academy of Management Perspectives, vol. 25, n ${ }^{\circ}$ 3, p. 6-18.

Chen M.-J. et Miller D. (2010). "West meets east: Towards an ambicultural approach to management", Academy of Management Perspectives, vol. 24, n 4, p. 17-24.

Cho J., Miller D. et Lee J. (2018). "Too much of a good thing: Family involvement and firm survival in listed Korean firms”, Journal of Family Business Strategy, vol. 9, no 4, p. 223-237.

Le Breton-Miller I. et Miller D. (2009). “Agency vs. stewardship in public family firms: A social embeddedness reconciliation”, Entrepreneurship Theory and Practice, vol. 33, p. 1169-1191.

Le Breton-Miller, I. et Miller, D. (2020). "Ideals-based accountability in select family firms", Journal of Business Ethics, vol. 163, no 2, p. 183-196.

Le Breton-Miller, I. et Miller, D. (2018). Alienation Among Management Scholars, HEC Montréal, Working paper.

Le Breton-Miller, I. et Miller, D. (2015). "The paradox of resource vulnerability: Considerations for organizational curatorship", Strategic Management Journal, vol. 36, $\mathrm{n}^{\mathrm{o}} 3$, p. 397-415. 
Miller D. et Le Breton-Miller I. (2017). "Underdog entrepreneurs: A challenge-based model of entrepreneurship", Entrepreneurship Theory \& Practice, vol. 41, n 1, p. 7-17.

Miller D. et Le Breton-Miller I. (2006). "Priorities, practices and strategies in successful and failing family businesses: An elaboration and test of the configuration perspective", Strategic Organization, November, vol. 4, n ${ }^{\circ}$, p. 379-407.

Miller D. et Le Breton-Miller I. (2005). "Management insights from great and struggling family businesses", Long Range Planning, vol. 38, p. 517-530.

Miller D., Le Breton-Miller I., Amore M., Minichilli A. et Corbetta G. (2017). "Institution allogics, family firm governance and performance", Journal of Business Venturing, vol. 32, $\mathrm{n}^{\mathrm{o}}$ 6, p. 674-693.

Miller D., Le Breton-Miller I. et Scholnick B. (2008). "Stewardship vs. stagnation: An empirical comparison of small family and non-family businesses", Journal of Management Studies, vol. 45, $\mathrm{n}^{\mathrm{o}} 1$, p. 50-78.

Miller D., Le Breton-Miller I. et Lester R.H. et Cannella A. (2007). “Are family firms really superior performers?”, Journal of Corporate Finance, vol. 13, p. 829-858.

Miller D., Lee J., Chang S. et Le Breton-Miller I. (2009). "Filling the institutional void: The social behavior and performance of family versus non-family technology firms in emerging markets”, Journal of International Business Studies, vol. 40, nº 5, p. 802-817.

Miller D. et Sardais C. (2011). "Angel agents: Agency theory reconsidered”, Academy of Management Perspectives, vol. 25, $\mathrm{n}^{\mathrm{O}} 2$, p. 6-13.

Miller D. (2018). "Challenging trends in configuration research: Where are the configurations?”, Strategic Organization, vol. 16, n 4, p. 463-469.

Miller D. (2011a). "The dyslexic researcher: A call to broadenour portals", Academy of Management Learning and Education, vol. 10, $\mathrm{n}^{\mathrm{o}} 2$, p. 340-350.

Miller D. (2011b). "Miller (1983) revisited: A reflection on EO research and some suggestions for the Future", Entrepreneurship Theory and Practice, vol. 35, n ${ }^{\circ}$ 5, p. 873-894.

Miller D. (1990). The Icarus Paradox: How Exceptional Companies Bring About Their Own Downfall, New York, NY: HarperCollins. (Traduit en français en 1992 sous le titre Le paradoxe d'Icare, Québec: Presses de l'Université Laval).

Miller D. et Xu X. (2019). "MBA CEOs, short-term management and performance”, Journal of Business Ethics, vol. 54, n ${ }^{\circ}$ 2, p. 285-300.

Miller D., Xu X. (2016). “A fleeting glory: Evidence of short-termism among celebrated MBA CEOs”, Journal of Management Inquiry, vol. 25, n 3, p. 286-300.

Misangyi V.F. et Acharya A.G. (2014). "Substitutes or complements? A configurational examination of corporate governance mechanisms", Academy of Management Journal, vol. 57, n ${ }^{\circ}$ 6, p. 1681-1705. 